\title{
Origin of the X-ray disc-reflection steep radial emissivity
}

\author{
J. Svoboda ${ }^{1}$, M. Dovčiak ${ }^{2}$, R. W. Goosmann ${ }^{3}$, P. Jethwa ${ }^{1}$, V. Karas ${ }^{2}$, G. Miniutti ${ }^{4}$, and M. Guainazzi ${ }^{1}$ \\ ${ }^{1}$ European Space Astronomy Centre of ESA, PO Box 78, Villanueva de la Cañada, 28691 Madrid, Spain \\ e-mail: jsvoboda@sciops.esa.int \\ 2 Astronomical Institute, Academy of Sciences, Boční II 1401, 14131 Prague, Czech Republic \\ 3 Observatoire astronomique de Strasbourg, Equipe Hautes Énergies, 11 rue de l'Université, 67000 Strasbourg, France \\ 4 Centro de Astrobiología (CSIC-INTA), Dep. de Astrofísica, ESA, PO Box 78, Villanueva de la Cañada, 28691 Madrid, Spain
}

Received 29 May 2012 / Accepted 25 July 2012

\begin{abstract}
Context. X-ray reflection off the accretion disc surrounding a black hole, together with the associated broad iron K $\alpha$ line, has been widely used to constrain the innermost accretion-flow geometry and black hole spin. Some recent measurements have revealed steep reflection emissivity profiles in a number of active galactic nuclei and X-ray binaries.

Aims. We explore the physically motivated conditions that give rise to the observed steep disc-reflection emissivity profiles.

Methods. We perform a set of simulations based on the configuration of a possible future high-resolution X-ray mission. Computations are carried out for typical X-ray bright Seyfert-1 galaxies.

Results. We find that steep emissivity profiles with $q \sim 4-5$ (where the emissivity is $\epsilon(r) \propto r^{-q}$ ) are produced considering either i) a lamp-post scenario where a primary compact X-ray source is located close to the black hole, or ii) the radial dependence of the disc ionisation state. If both effects are taken into account, emissivity profiles as steep as $q \sim 7$ can be obtained from X-ray spectra modelled via conventional reflection models. We also highlight the role of the reflection angular emissivity: the radial emissivity index $q$ is overestimated when the standard limb-darkening law is used to describe the data.

Conclusions. Very steep emissivity profiles with $q \geq 7$ are naturally obtained by applying reflection models that take into account the radial profile $\xi(r)$ of the disc ionisation induced by a compact X-ray source located close to the central black hole.
\end{abstract}

Key words. black hole physics - accretion, accretion disks - relativistic processes - galaxies: nuclei

\section{Introduction}

The innermost black-hole accretion discs of active galactic nuclei (AGNs) and black hole binaries may be revealed through their X-ray radiation released as either the thermal radiation of the disc (Zhang et al. 1997; McClintock \& Remillard 2006) or the result of the inverse Compton scattering of the thermal photons on the relativistically moving electrons in the hot corona above the disc (Shapiro et al. 1976; Haardt \& Maraschi 1991). While the first is relevant only to stellar-mass black holes where the accretion disc is heated to very high temperatures (around $10^{7} \mathrm{~K}$ ), the second is common for any accreting black hole over the entire possible mass scale.

Some fraction of the photons scattered in the corona reflect off the disc surface before reaching the observer. This socalled reflection spectrum is characterised by a Compton hump at energies of around $20-40 \mathrm{keV}$, fluorescent lines of which the iron $\mathrm{K} \alpha$ line at $6.4-6.97 \mathrm{keV}$ is the most prominent, and a soft excess below $1 \mathrm{keV}$. The overall spectral shape depends on the ionisation of the disc surface. The spectrum is then smeared by the relativistic effects including energy shift, aberration, and light-bending. The inner disc radius, inclination angle, average ionisation state, and reflection-emissivity radial profile can in principle be determined from the resulting X-ray spectral shape. The inner disc radius plays a particularly important role. As shown e.g. by Reynolds \& Fabian (2008), the inner-disc reflection radius can be associated with the innermost stable circular orbit, which only depends on the black hole spin. Hence, $\mathrm{X}$-ray reflection spectra can be used to estimate the black hole spin in both AGN and black hole binaries (see e.g. Reynolds \& Nowak 2003; Miller et al. 2009; Brenneman \& Reynolds 2009, and references therein).

The black hole spin measurements are thus influenced by the geometry of the disc-illuminating corona and the local properties of the disc that affect the re-processing and re-emission of the incident photons. Current relativistic kernels that are applied to reflection (and/or iron line) models to include the relativistic effects on the spectral shape (Laor 1991; Dovčiak et al. 2004; Beckwith \& Done 2004; Brenneman \& Reynolds 2006) are based on a series of simplified assumptions and, in particular, they assume a single/broken power-law form of the radial reflection emissivity and an angular emissivity law defined by a simple analytical formula, most frequently employing a limbdarkening profile (Laor 1991).

In Svoboda et al. (2009), we pointed out that the choice of a particular angular emissivity law can affect the derived relativistic parameters, including the black hole spin. In the present work, we extend our analysis to the radial emissivity law and, in particular, we investigate the physical conditions under which steep radial emissivity profiles are produced by considering the effects of (i) primary X-ray source location, (ii) radial disc ionisation profile, and (iii) angular emissivity law.

The intrinsic disc emissivity is naturally expected to decrease with increasing distance, i.e. the reflection emissivity is

$\epsilon(r)=r^{-q}$,

where $q$ is the emissivity index that can be constant over all radii or a varying quantity. The thermal dissipation of the disc 
decreases as $r^{-3}$ (Shakura \& Sunyaev 1973; Novikov \& Thorne 1973). Therefore, the simplest assumption is postulating the same dependence for the reflection. The more energetic photons are injected into the innermost regions, and so, more intense irradiation of the disc occurs there. In addition, assuming a pointlike X-ray source at height $h$ on the disc axis, the irradiation of the disc in the absence of any relativistic effect is proportional to $\left(r^{2}+h^{2}\right)^{-3 / 2} \propto r^{-3}$, as shown e.g. by Reynolds \& Begelman (1997). An emissivity profile with $q=3$ is therefore considered as standard, while steeper/flatter indices may need to be explained.

Steep emissivity profiles have been reported from the analysis of the X-ray spectra of other AGN, such as MCG-6-30-15 (Fabian et al. 2002; Vaughan \& Fabian 2004; Miniutti et al. 2007), 1H0707-495 (Fabian et al. 2009; Zoghbi et al. 2010; Wilkins \& Fabian 2011; Dauser et al. 2012), and IRAS 132243809 (Ponti et al. 2010), as well as black hole binaries, such as XTE J1650-500 (Miniutti et al. 2004), GX 339-4 (Miller 2007), and Cyg X-1 (Fabian et al. 2012). The measured indices reach values up to $q \approx 7$.

To provide a physical picture of the steep radial emissivity in MCG-6-30-15, Wilms et al. (2001) invoke strong magnetic stresses that should act in the innermost region of the system. This should correspond to the enhanced dissipation of a considerable amount of energy in the accretion disc at small radii. If the magnetic field lines thread the black hole horizon, the dissipation could be triggered by the magnetic extraction of the black hole rotational energy, perhaps via the Blandford-Znajek effect (Blandford \& Znajek 1977), but it could be also supplemented by a rather efficient slowing of the rotation, as also seen in recent GRMHD simulations (e.g. Penna et al. 2010). The efficiency of the competing processes still needs to be assessed.

Martocchia et al. (2000) examined whether the required steep emissivity law as well as the predicted equivalent width of the cold reflection line of iron and the Compton reflection component can be reproduced in a phenomenological (lamp-post) model where the X-ray illuminating source is located on the common symmetry axis of the black hole and the equatorial accretion disc. They suggested that the radial emissivity function of the reflection component steepens when the height parameter of the primary irradiation source decreases. The enhanced anisotropy of the primary X-rays was identified as a likely agent acting in this process. The emissivity in the XMM-Newton spectrum of MCG-6-30-15 was successfully reproduced by adopting this lamp-post geometry (Martocchia et al. 2002; Miniutti et al. 2003; Niedźwiecki \& Życki 2008).

To explain the steep radial emissivity, we explore several simple test models and analyse them with the simulated data. The paper is organised as follows. Section 2 describes the data generation. The effects of the lamp-post geometry of the corona, the angular directionality, and the radially stratified ionisation on the radial emissivity are discussed in Sects. 3-5, respectively. An example of the combined effect is presented in Sect. 6. The obtained results are discussed in Sect. 7 and summarised in Sect. 8.

\section{Data simulation}

Our goal is to build a series of physically motivated reflection models and to examine the radial emissivity law that would be inferred from deep, high-quality X-ray spectral observations of AGN and/or black hole X-ray binaries with either current or future X-ray facilities. We build theoretical reflection spectral models that are then put into X-ray spectral simulations to produce high quality X-ray data. The simulated data are then modelled with the currently available disc-reflection models and standard spectral tools. To evaluate the possibility of measuring the simulated effects with future X-ray missions, we adopted a preliminary response matrix for the Athena X-ray mission (Nandra 2011). This ensures that our results are not driven/limited by the signal-to-noise that can be reached with current X-ray observatories.

We performed our spectral analysis within the $2-10 \mathrm{keV}$ energy range where one of the most prominent reflection feature, the iron $\mathrm{K} \alpha$ line, occurs. The preliminary response matrix ${ }^{1}$, was re-binned by a factor of ten between channels 2700 and $8800(2-10 \mathrm{keV})$. The other channels were not used. This provided us with sufficient spectral resolution and increase in the computational speed. We used Xspec (Arnaud 1996), version 12.6.0ab for the spectral fitting. We used the most recent version of the KY code (Dovčiak et al. 2004), which includes the lamp-post geometry (Dovčiak et al., in prep.). The flux of the model was chosen to be similar to that of bright Seyfert galaxies, i.e. $\approx 3 \times 10^{-11} \mathrm{erg} \mathrm{cm}^{-2} \mathrm{~s}^{-1}$ (Nandra et al. 2007). The simulated observation time was $100 \mathrm{ks}$, which is comparable to the average exposure time of AGNs observed with the current X-ray satellites.

\section{Lamp-post scheme}

We first investigate how the radial emissivity depends on the geometry of the corona. If the corona is localised the illumination of the disc decreases with the growing distance from the source in a way determined by the position of the corona and by the gravitational field of the central black hole. The configuration when the corona is very compact and located just above the black hole, known also as the lamp-post scheme, was studied as a simple disc-corona scenario by Matt et al. (1991) and George \& Fabian (1991). In a physical picture, the source above the black hole can be imagined, e.g., as the base of a jet.

In this scenario, the irradiation far from the source radially decreases as $r^{-3}$. In the central region, the relativistic effects influence the disc illumination, thus shape the reflection spectra of black hole accretion discs (Miniutti \& Fabian 2004). As a result, the different parts of the disc are irradiated with different intensities, making the emissivity profiles in reflection models distinct from the standard value of $q=3$. If the height of the source is sufficiently close to the black hole event horizon, light bending implies higher irradiation of the innermost region compared to the outer parts of the disc.

The exact profile of the radial emissivity depends on the geometrical properties of the source. Different cases of axial, orbiting, jet, and extended sources were studied by Wilkins \& Fabian (2012). The steepest profiles were obtained for point-like sources at small heights along the vertical axis. In the most extreme scenario, when the source is moving towards the black hole, the Doppler boosting might increase this effect. However, there is no observational evidence for such an inflow of the matter perpendicular to the disc plane, while outflows in the form of a jet or a disc wind are observed in many sources (e.g. Merloni et al. 2003; Blustin et al. 2005; Krongold et al. 2007; Tombesi et al. 2010).

In the following, we consider the stationary lamp-post source and investigate the radial emissivity profile of the disc reflection radiation for different heights of the source. The physical set-up of the model is a combination of the general-relativistic lamp-post scheme for an X-ray illuminated accretion disc near a

1 ftp://ftp.rssd.esa.int/pub/athena/09052011_Responses 
Table 1. Inner radial emissivity index $q_{\mathrm{in}}$ and the break radius $r_{\mathrm{b}}$ inferred for a different height and directionality in the lamp-post model.

\begin{tabular}{l|cc|cc|cc|cc}
\multicolumn{10}{c|}{$a=0.94$} \\
\hline \hline & \multicolumn{2}{|c|}{ Numerical } & \multicolumn{2}{c|}{ Limb brightening } & \multicolumn{2}{c}{ Isotropic } & \multicolumn{2}{c}{ Limb darkening } \\
$h\left[r_{g}\right]$ & $q_{\text {in }}$ & $r_{\mathrm{b}}$ & $q_{\text {in }}$ & $r_{\mathrm{b}}$ & $q_{\text {in }}$ & $r_{\mathrm{b}}$ & $q_{\text {in }}$ & $r_{\mathrm{b}}$ \\
\hline 1.5 & $4.72_{-0.08}^{+0.07}$ & $6.1_{-0.2}^{+0.2}$ & $4.54_{-0.07}^{+0.06}$ & $6.4_{-0.2}^{+0.2}$ & $4.96_{-0.08}^{+0.07}$ & $6.0_{-0.2}^{+0.3}$ & $5.29_{-0.07}^{+0.06}$ & $5.7_{-0.1}^{+0.2}$ \\
3.0 & $3.3_{-0.1}^{+0.1}$ & $6.3_{-1.1}^{+1.9}$ & $3.2_{-0.2}^{+0.3}$ & $8.0_{-2.6}^{+3.4}$ & $3.2_{-0.1}^{+0.1}$ & $15_{-3}^{+10}$ & $3.3_{-0.1}^{+0.1}$ & $20_{-5}^{+0}$ \\
10 & $1.3_{-0.2}^{+0.1}$ & $16_{-1}^{+1}$ & $2.3_{-0.1}^{+0.1}$ & $55_{-10}^{+9}$ & $2.3_{-0.1}^{+0.2}$ & $48_{-7}^{+5}$ & $2.5_{-0.1}^{+0.1}$ & $60_{-15}^{+15}$ \\
\hline
\end{tabular}

rotating black hole (Martocchia et al. 2000) and a self-consistent Monte Carlo scheme for the X-ray reprocessing within the disc environment (Goosmann et al. 2006). We used local (re-) emission tables that were computed by the radiative transfer code NOAR (Dumont et al. 2000, Sect. 5) for the case of "cold" reflection (i.e. for neutral or weakly ionised matter). Photoabsorption, Compton scattering, and the fluorescent emission of the iron $\mathrm{K}$ line are considered. A stratified plane-parallel atmosphere irradiated by a power law with the photon index $\Gamma=1.9$ is assumed. A large number of primary photons were sampled in the $2-300 \mathrm{keV}$ energy range to ensure sufficiently high quality statistics. At all local emission angles, the Poissonian noise level is much smaller than any relevant spectral feature. The computations were done for various incident local emission angles, both polar and azimuthal.

In the present paper, we employ a new implementation of the lamp-post scenario that is based on the KY package (Dovčiak et al. 2004, a more detailed description of the new model is presented in the parallel paper Dovčiak et al., in prep.). All the relativistic effects on the photon energies and trajectories, such as aberration and light bending, are incorporated in the model. Here, we study whether it is possible to approximate the radial emissivity of the lamp-post model by a simplified profile in the form of a broken power-law, as usually done in current modelling of the data.

For this purpose, we generated data as described in Sect. 2 for a power-law model with photon index $\Gamma=1.9$ and the relativistic line in the lamp-post geometry described above. The spin parameter was set to $a=0.94$ (in the geometrised units where $c=G=M=1$ ). The other parameters are the inclination angle of the disc $i=30 \mathrm{deg}$, which is a typical value for the inclination of Seyfert 1 galaxies (de La Calle Pérez et al. 2010), the inner radius coinciding with the marginally stable or$\operatorname{bit}\left(r_{\mathrm{in}}=r_{\mathrm{ms}}\right)$ and the outer radius corresponding to $r_{\mathrm{out}}=400 r_{\mathrm{g}}$, where $r_{\mathrm{g}} \equiv \frac{G M}{c^{2}}$ is the gravitational radius. We considered three cases of the source height, $h=1.5,3$, and $10 r_{\mathrm{g}}$.

We then fitted the radial emissivity profile resulting from the simulations with a broken power-law model in which the emissivity index is $q_{\text {in }}$ within a break radius $r_{\mathrm{b}}$ and $q_{\text {out }}=3$ at larger distances. As expected, we found that $q_{\text {in }}$ increases as the source height $h$ decreases, while $r_{\mathrm{b}}$ decreases. Steep emissivity profiles in the inner regions (i.e. $q_{\text {in }} \geq 3$ ) were only obtained for $h \lessgtr 3 r_{\mathrm{g}}$, as summarised in Table 1 in the column labelled "numerical".

\section{Interplay between the radial and angular emissivity profiles}

When fitting the data, the local intensity of the re-processed radiation emitted from the disc is often assumed to be divided into two separate parts - the radial and angular dependence. The latter one characterises the emission directionality. However, owing to the large rotational velocity of the disc and the strong gravity near the black hole, the photons that reach the observer are emitted under different angles at different locations. Therefore, the angular part of the emissivity depends on radius as well, and the above separation is invalid. The relativistic effects, namely aberration and light bending, cause the emission angle in the innermost region to always be very high (almost 90 degrees with respect to the disc normal) - see Appendix C in Dovčiak (2004), or Fig. 3 in Svoboda et al. (2009). Although it is not an axisymmetric problem, the almost radial decrease in the emission angle is readily apparent, which invokes the link between the radial and angular emissivity (Beckwith \& Done 2004; Svoboda et al. 2009).

To show the impact of the directionality on the radial emissivity profile, we considered the cases of

1. our numerical computations;

2. limb brightening $I\left(\mu_{\mathrm{e}}\right) \approx \ln \left(1+\mu_{\mathrm{e}}^{-1}\right)$ (Haardt 1993);

3. isotropic emissivity;

4. limb darkening $I\left(\mu_{\mathrm{e}}\right) \approx 1+2.06 \mu_{\mathrm{e}}($ Laor 1991),

where $\mu_{\mathrm{e}}$ is the cosine of the local emission angle. The simulated data were created with our numerical model of the directionality integrated over all incident angles. The NOAR code (Dumont et al. 2000) was employed in the calculations. Free-free absorption, the recombination continua of hydrogen- and helium-like ions, and both direct and inverse Compton scattering were taken into account.

Figures 1 and 2 show contour plots of the radial emissivity index and the spin, and the break radius, respectively, for different prescriptions of the angular directionality. The lamp-post model with the source height at $h=1.5 r_{\mathrm{g}}$ was used in the data simulation, while the fitting model employed a broken powerlaw emissivity. In the contour calculations, only the two parameters of interest were allowed to vary. The others were fixed to their default or best-fit (in the case of break radius) values. The best-fit parameters are summarised in Table 1.

The worst fit was obtained for the model with limb darkening for which significantly steeper values of the radial emissivity were required. This emissivity law is widely used in the reflection models. However, it somewhat contradicts several models of X-ray illuminated disc atmospheres (Ghisellini et al. 1994; Życki \& Czerny 1994; Goosmann et al. 2006; Różańska et al. 2011). Its application causes an appreciable underestimation of the innermost flux. The fit therefore requires an artificially steep value of the radial emissivity index in order to compensate for this loss of counts from the central region where the emission angle is grazing.

\section{Radially structured ionisation of the disc}

The interplay between the radial and angular emissivity shows that the steep radial emissivity in the observational data might be caused by an invalid model assumption. Yet, there is another frequently used assumption in the reflection scenario that can 

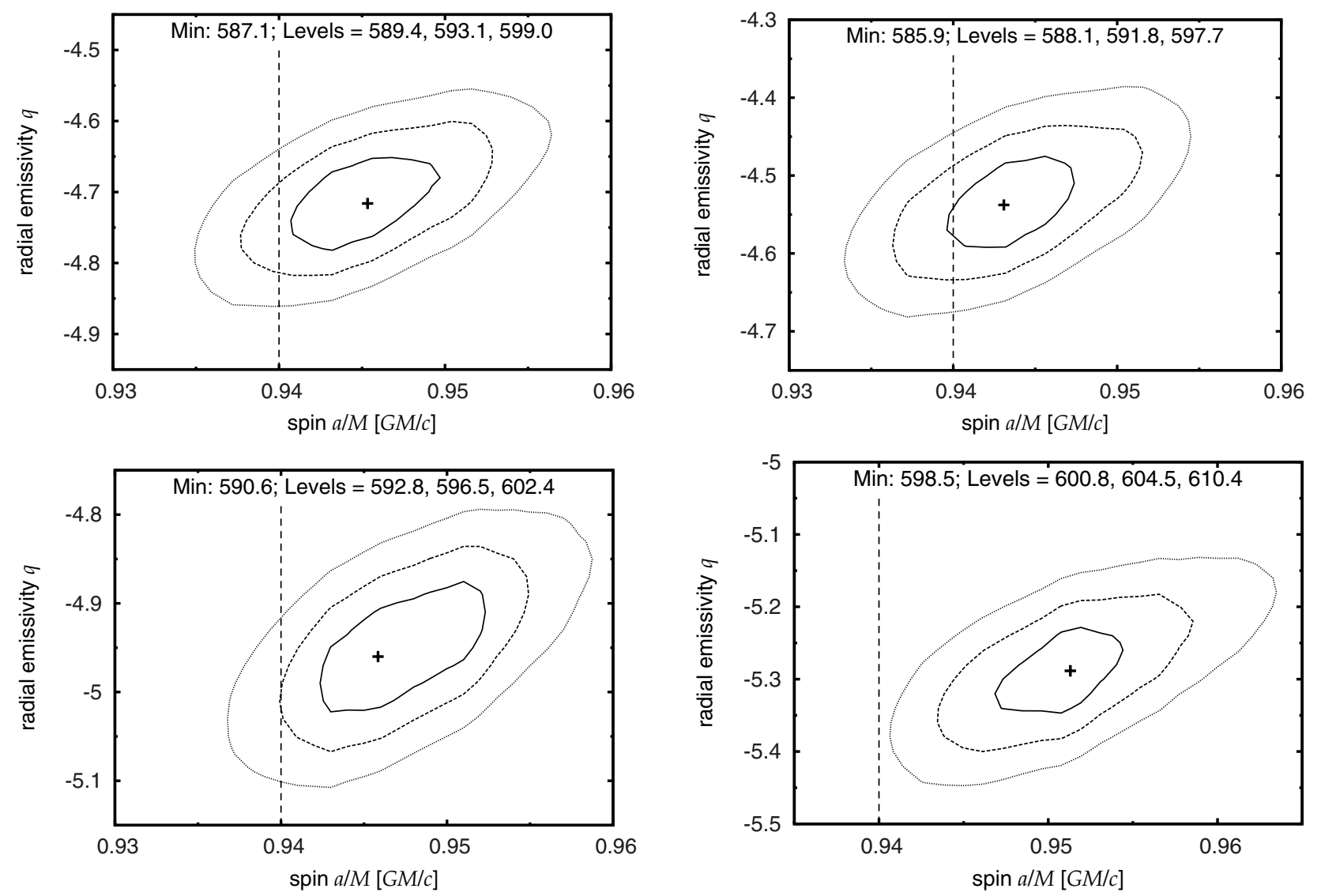

Fig. 1. Contour plots of the spin $a$ and the radial emissivity parameter $q$. The data were generated with the lamp-post model with the height $h=$ $1.5 r_{\mathrm{g}}$. The default value of the spin was $a=0.94$, which is indicated by a dashed line in the graph. Different prescriptions for the angular emissivity were used. Top left: angular emissivity from numerical calculations. Top right: limb brightening. Bottom left: isotropic. Bottom right: limb darkening. The $\chi^{2}$ values corresponding to the best fit (indicated by a small cross) and to the $1 \sigma, 2 \sigma$, and $3 \sigma$ levels are shown in the text legend.

contribute to this effect as well - the constant ionisation over the whole surface of the disc. The intensity of the disc irradiation, whether approximated by a (broken) power-law decrease or a lamp-post illumination in curved space-time, decreases with radius. Therefore, the ionisation of the disc surface may respond accordingly (see e.g. Matt et al. 1993).

The importance of the photoionisation of the disc surface in AGNs was studied by several authors (Zycki et al. 1994; Nayakshin et al. 2000; Ballantyne et al. 2001, 2011). Ballantyne et al. (2003) interpreted the X-ray reflection spectrum of MCG 6-30-15 as being composed of a highly ionised reflection from the innermost region and a cold one from the outermost accretion disc. The radially dependent ionisation in the existing data was also discussed by Zhou et al. (2011). The photoionisation was also suggested as a possible explanation of the non-detection of the spectral imprints of the relativistically smeared reflection in some sources (Reynolds et al. 2004; Svoboda et al. 2010; Bhayani \& Nandra 2011; Brenneman et al. 2012).

In general, the ionisation of the disc surface depends on several other physical quantities such as the density, vertical structure, and thermal heating (see e.g. Nayakshin \& Kallman 2001; Różańska et al. 2002; Goosmann et al. 2007, and references therein). In particular, Nayakshin et al. (2000) showed that considering hydrostatic and ionisation balance and radiative transfer in a plane-parallel geometry resulted in a cold core within the accretion disc and highly ionised outer layers. A detailed description of the disc ionisation is beyond the scope of this paper. Here, we simply assume that the radial dependence of the ionisation may be relevant, as a natural consequence of the radial dependence of the disc illumination by the primary radiation.

If the photoionisation is the dominating factor determining the state of the plasma the ionisation parameter can be defined as (see e.g. Ross \& Fabian 1993)

$\xi=\frac{4 \pi F_{\mathrm{inc}}}{n_{\mathrm{H}}}$,

where $F_{\text {inc }}$ is the incident (irradiation) flux and $n_{\mathrm{H}}$ is the number density of hydrogen. Solar abundances are assumed for the other elements. Typically, X-ray spectra are fitted using only one reflection component, i.e. assuming $\xi$ constant over the whole disc. However, in general, Eq. (2) may be re-written as

$\xi=\frac{4 \pi F_{\mathrm{inc}}(r)}{n_{\mathrm{H}}(r)}$,

with the radial dependence of the irradiating flux and the density.

Figure 3 shows the radial dependence of the ionisation for different density profiles: constant disc density, Novikov-Thorne (Novikov \& Thorne 1973), and a profile from general relativistic magneto-hydrodynamic simulations by Hawley \& Krolik (2006) for isotropic irradiation decreasing like $F_{\text {inc }} \propto r^{-3}$. In all cases, the ionisation decreases with the radius according to a decreasing strength of the irradiation. Although an unknown density profile introduces an uncertainty in the radial ionisation description, its effect is, however, not as strong a function of the radius as the irradiation profile itself. We therefore assumed a constant density over the whole disc surface in our more detailed analysis. 
J. Svoboda et al.: Origin of the X-ray disc-reflection steep radial emissivity
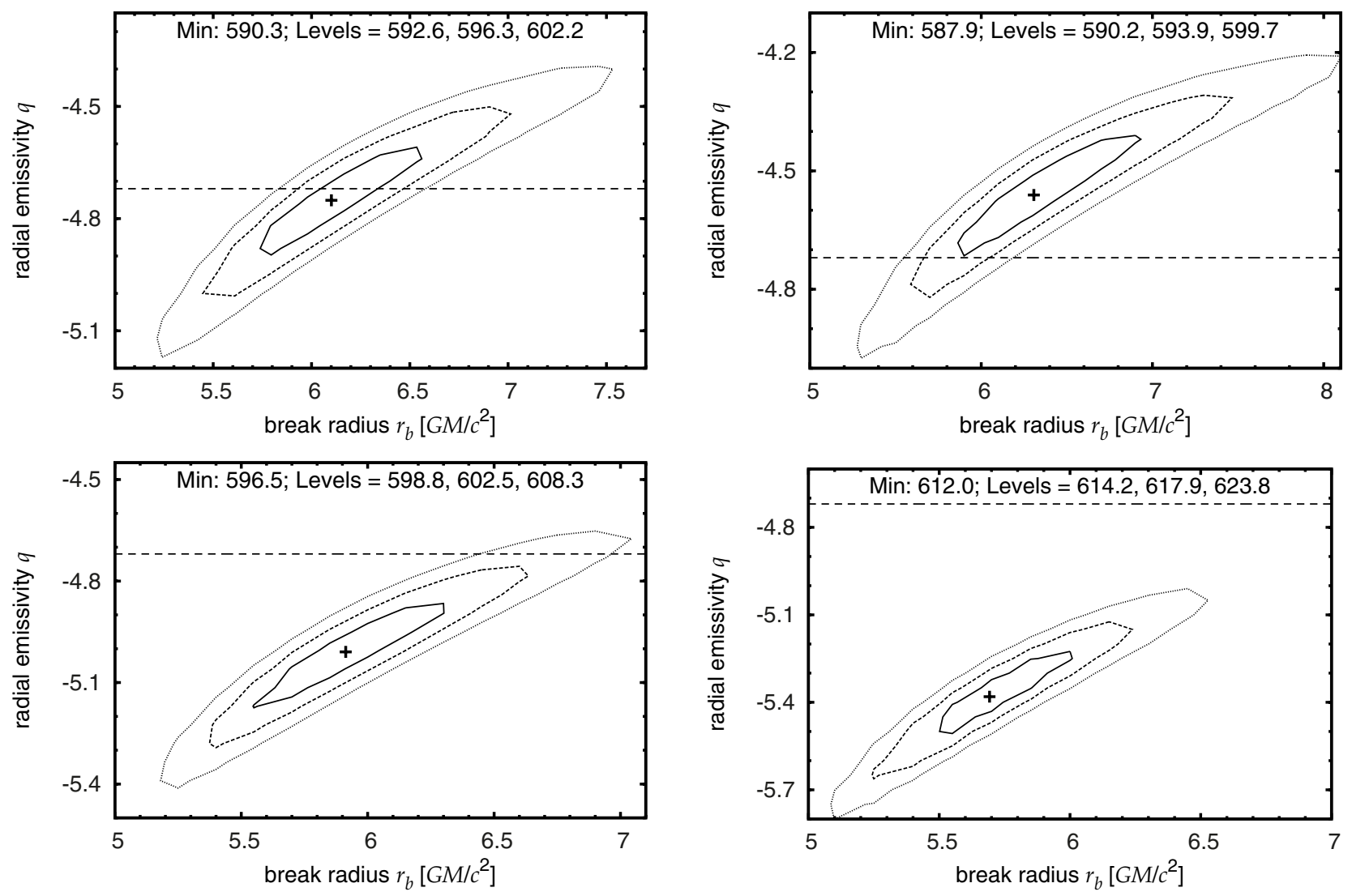

Fig. 2. Contour plots of the radial emissivity and the break radius for the height $h=1.5 r_{\mathrm{g}}$. The legend is the same as in Fig. 1. A horizontal dashed line representing the best-fit value from Table $1, q=4.72$, is in the graph only for comparison.

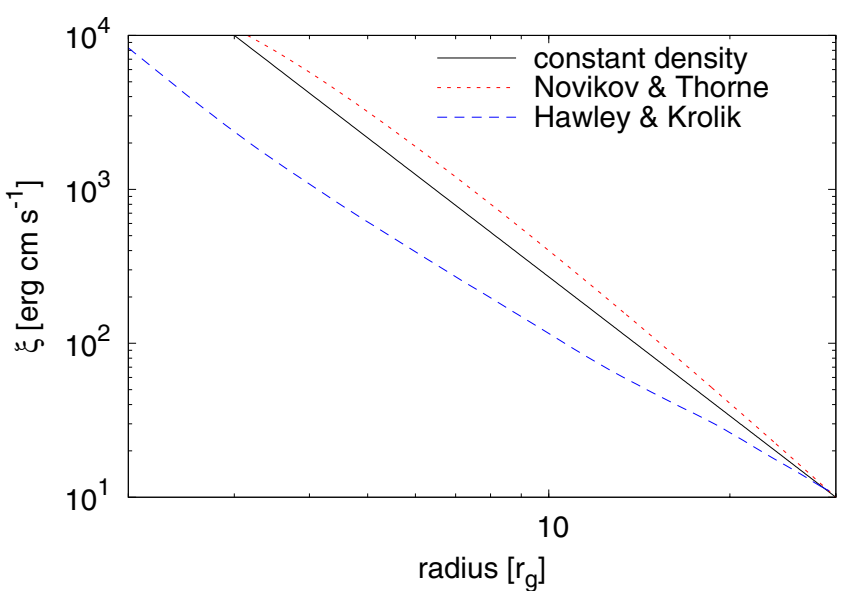

Fig. 3. Radial dependence of the ionisation parameter for different prescriptions of the density: constant (black, solid), Novikov-Thorne profile (red, dotted), and from the GRMHD simulations by Hawley \& Krolik (2006) (blue, dashed). The assumed irradiation is $F_{\text {inc }} \propto r^{-3}$. The considered spin value is $a=0.99$.

To describe the ionisation profile, a specific ionisation parameter has to be set at a given radius. In general, this normalisation depends on the luminosity and the distance of the irradiating source, in addition to the density of the disc. It is therefore different for different kinds of sources, and moreover, depends on the variable accretion rate. The choice of the normalisation factor is thus somewhat arbitrary for our purposes. Here we assumed that the ionisation of the disc is $\xi=10 \mathrm{ergs} \mathrm{cm} \mathrm{s}^{-1}$ at $30 r_{\mathrm{g}}$ since the neutral iron line is observed in most spectra of AGNs.
We simulated a spectrum produced under these conditions by dividing the disc into discrete rings of fixed $\xi$ and summing the spectra produced in each ring. To generate these spectra, we used a grid of REFLIONX models (Ross \& Fabian 2005) with a different ionisation state convolved with the KY model (Dovčiak et al. 2004), corresponding to different emission regions across the disc. For each ring, the generated reflection component has equal integrated flux and the radial emissivity is governed by the relativistic convolution models. The innermost radius coincides with the marginally stable orbit, and the outer radius was set to $400 r_{\mathrm{g}}$. The spin value was chosen to be $a=0.94$, i.e. $r_{\mathrm{ms}} \approx$ $2 r_{\mathrm{g}}$. The inclination angle was chosen to be $30 \mathrm{deg}$. The photon index of the primary power-law radiation and its normalisation were set to $\Gamma=2.0$ and $K_{\Gamma}=10^{-3}$. The standard value, $q=3$, was adopted for the radial emissivity index. We used solar iron abundances.

The total reflection fraction to the primary radiation corresponds to the case of an isotropically irradiated disc, i.e. similar to that of $R \approx 1$ in the PEXRAV model (Magdziarz \& Zdziarski 1995). Such a constructed model, which we henceforth refer to as a "complex" model, is shown with its components in Fig. 4. The straight dotted line in the plot corresponds to the powerlaw continuum irradiating the disc. The iron line around $6 \mathrm{keV}$ may be used as a diagnostic of the individual reflection components. The cold reflection from the outer disc (13.9-400 $\left.r_{\mathrm{g}}\right)$ corresponds to the most prominent peak. The second largest peak is produced by the ring occurring at $6.3-13.9 r_{\mathrm{g}}$. The components from the more central regions have more smeared profile and more red-shifted iron lines. The disc separation into individual rings was done according to the gradient of the ionisation parameter, so the innermost rings are much narrower than the 
A\&A 545, A106 (2012)

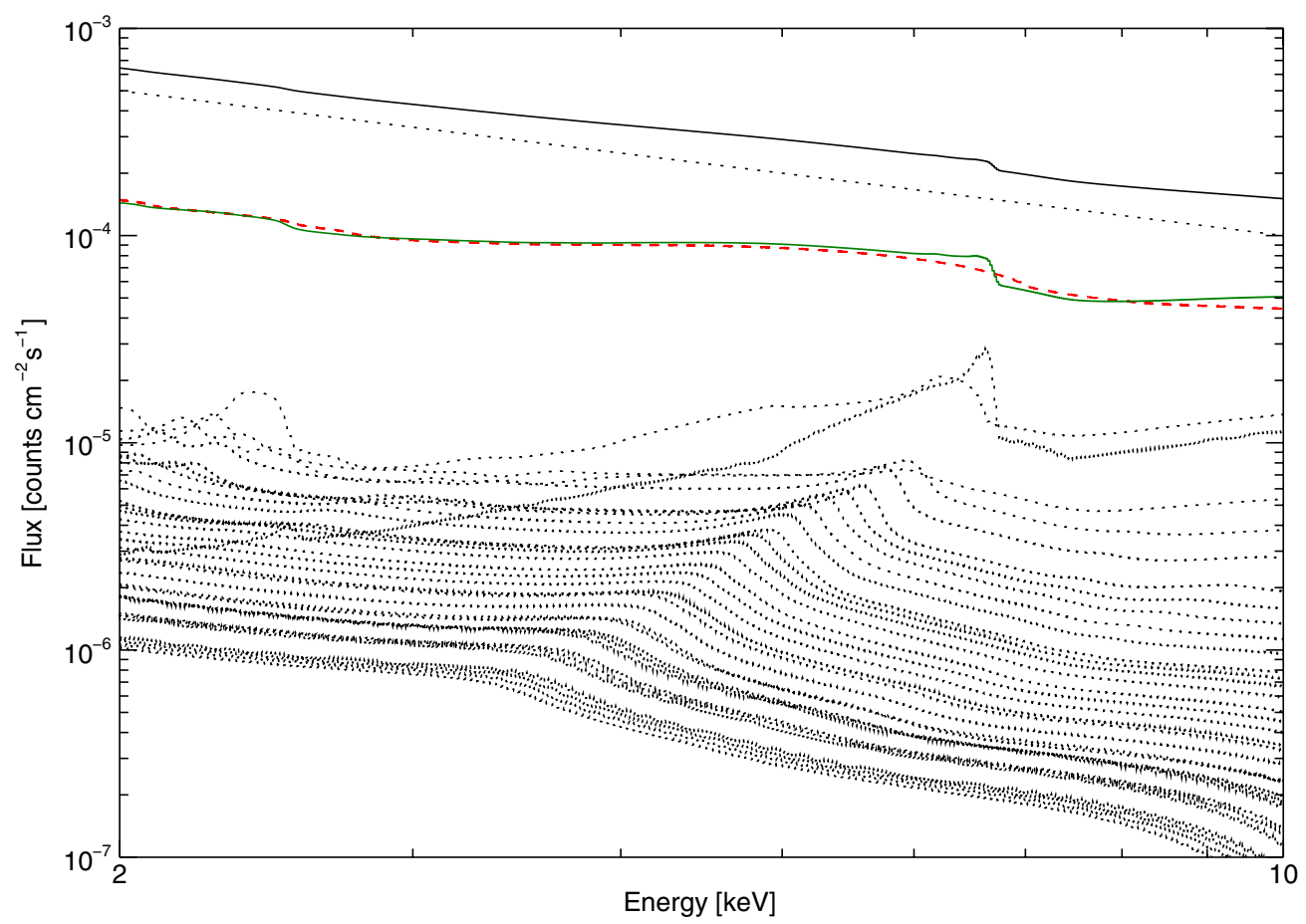

Fig. 4. Plot of the "complex" model. The top solid line shows the total model flux, while the dotted lines are its components. The sum of all reflection components is shown by the green solid line. The red dashed line represents the best-fit reflection model assuming single ionisation. See the main text for more details.
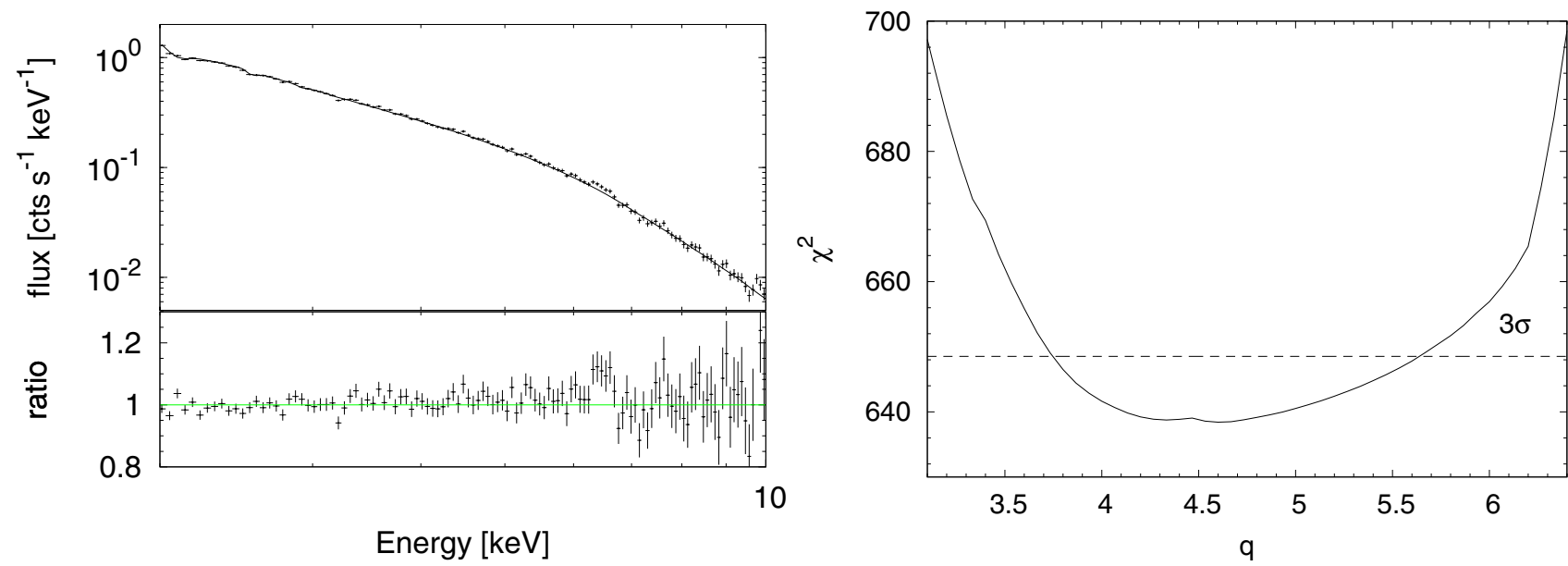

Fig. 5. Left: data created by the "complex" model (see Fig. 4) and their ratios with respect to the single-ionisation reflection model. Right: dependence of the fit-goodness on the radial emissivity parameter of the single reflection model. The dashed line corresponds to a $3 \sigma$ confidence level.

outer parts and thus contribute less to the total flux (the bottom lines in the graph). The total reflection component is shown by a green line in the plot.

We generated the data in the same way as described in Sect. 2 . We then fitted the data in the $2-10 \mathrm{keV}$ energy range with a model consisting of only a power-law continuum and a single reflection component with a broken power-law radial emissivity. The inner radial emissivity index, the break radius, the ionisation, and the normalisation of the reflection model were the only parameters that were allowed to vary during the fitting procedure.

The resulting single-ionisation model represents a relatively good fit to the simulated data with $\chi^{2} / v=639 / 606 \approx 1.05$, but with significant discrepancies in the iron line and its edge. The model (indicated by a red line) is compared with the "complex" reflection in Fig. 4. An apparent difference occurs at the iron line, where a peak from distant cold reflection is missing in the singleionisation model. The data residuals from this model are shown in the left panel of Fig. 5. The fit improved by $\Delta \chi^{2}=16$ when a Gaussian line was added to the model. The energy and the width of the additional line were found to be $E=6.45_{-0.07}^{+0.08}$ and $\sigma=$ $0.13_{-0.05}^{+0.07}$. The equivalent width is $41 \pm 17 \mathrm{eV}$.

The best-fit values of the reflection model parameters are summarised in the middle column of Table 2. The value of the ionisation parameter is around $250 \mathrm{erg} \mathrm{cm} \mathrm{s}^{-1}$. The radial emissivity index is required to be significantly steeper, $q \geq 4$, as it is also clearly visible in the right panel of Fig. 5. Figure 6 (left panel) shows a contour plot between the radial emissivity parameter and the break radius. A preferred value of the break radius is larger than $10 r_{\mathrm{g}}$, which demonstrates the significance of the emissivity steepening (i.e. that it is not related only to a narrow innermost ring). The fit is insensitive to larger values than 

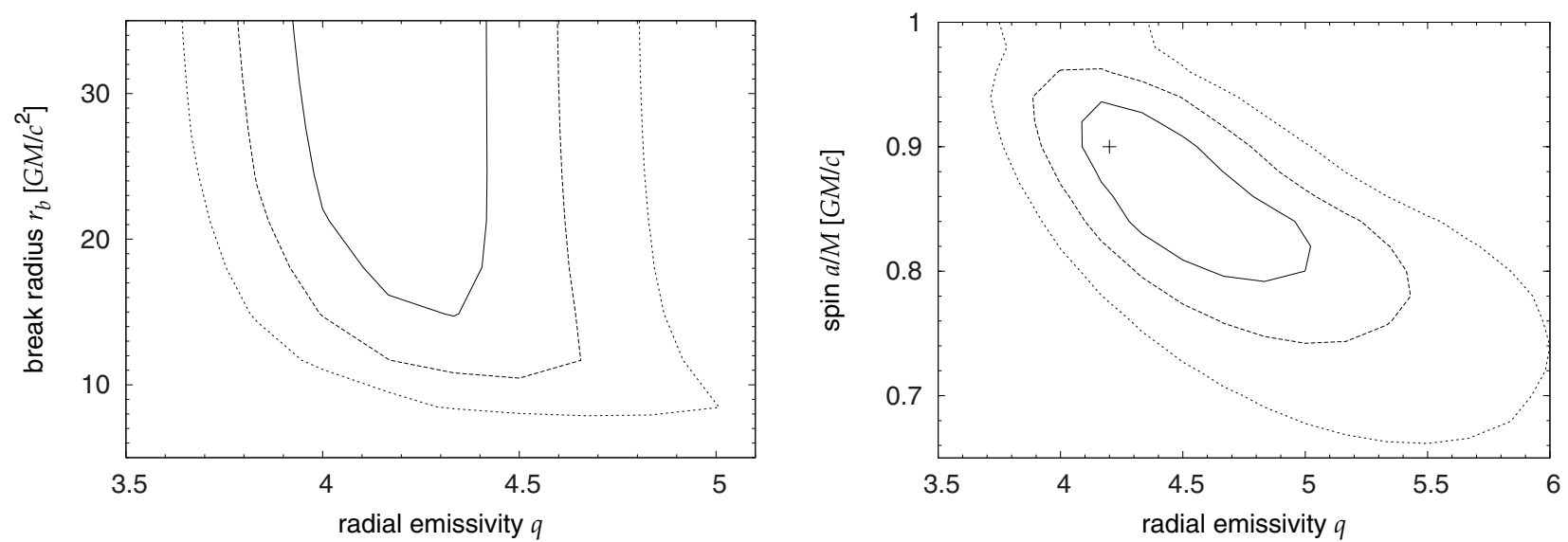

Fig. 6. Contour plots of the radial emissivity parameter $q$ and the break radius $r_{\mathrm{b}}(l e f t)$ and the spin $a$ (right), respectively. Data were created by a "complex" model with radially stratified ionisation, isotropic irradiation, and angular emissivity. A single-ionisation model with power-law radial emissivity was used to fit the data. The individual contours correspond to $1 \sigma, 2 \sigma$, and $3 \sigma$ level, respectively.
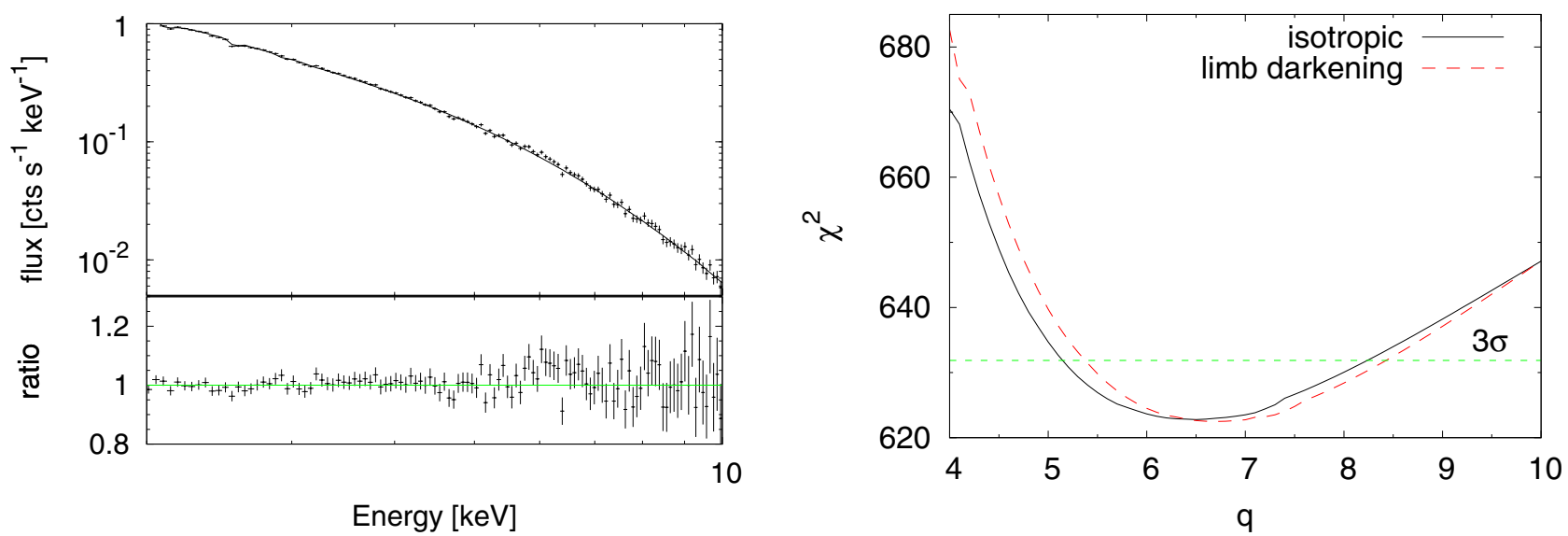

Fig. 7. As in Fig. 5, but the data were created by a lamp-post model with the height $h=1.5 r_{\mathrm{g}}$. In the right panel, we show a further overestimation of $q$ by employing limb darkening instead of isotropic angular directionality used in the data simulation (red dashed line).

Table 2. Resulting parameter values of the single reflection model applied to the data simulated by a "complex" reflection model.

\begin{tabular}{l|c|c}
\hline \hline Parameter & Isotropic & $h=1.5 r_{\mathrm{g}}$ \\
\hline Spin & $0.86_{-0.07}^{+0.08}$ & $0.94 \pm 0.02$ \\
Inner radial emissivity & $4.5_{-0.5}^{+0.6}$ & $6.7_{-0.8}^{+0.9}$ \\
Ionisation [ergs cm s ${ }^{-1}$ ] & $250_{-20}^{+30}$ & $230_{-10}^{+20}$ \\
\hline Fit goodness $\chi^{2} / v$ & $639 / 606$ & $623 / 606$ \\
\hline
\end{tabular}

Notes. Two cases of assumed irradiation profile are considered, isotropic with the radial emissivity index $q=3$, and a point source at the height $h=1.5 r_{\mathrm{g}}$. Default value of the spin was $a=0.94$.

about $20 r_{\mathrm{g}}$. A contour plot between the radial emissivity parameter and the spin is shown in the right panel of Fig. 6. A mutual degeneration of these two parameters is evident from the plot. However, significantly steeper values of the radial emissivity are required for any reasonable value of the spin.

\section{Steep radial emissivity due to the combined effect}

We have presented three different effects that may account for a steep radial emissivity. However, they are not completely independent and the most likely scenario is that they occur together. We therefore simulated the data by considering all these effects together. We considered a lamp-post scenario with a very low height, $h=1.5 r_{\mathrm{g}}$. We calculated the ionisation profile of a constant-density disc produced by this irradiation taking into account all the relativistic effects such as photon light-bending and aberration. For simplicity, we assumed an isotropic directionality, i.e. that the intensity of reflected radiation does not locally depends on the emission angle.

The data were then fitted by a single-ionisation reflection model with the radial emissivity defined by a broken power-law with the index $q$ as a free parameter within $30 r_{\mathrm{g}}$ and $q=3$ beyond this radius. The spectrum is reflection-dominated due to the strong light-bending effect (Miniutti \& Fabian 2004). The parameters of the primary power-law were fixed. The resulting model describes the data quite well with $\chi^{2} / v=623 / 606 \approx$ 1.03 , again with some residuals in the iron-line energy band (see the left panel of Fig. 7). The best-fit values are summarised in the last column of Table 2.

The radial emissivity is very steep, $q \approx 7$. We show the dependence of the fit goodness on this parameter in the right panel of Fig. 7, where we compare two cases of assumed directionality. A value larger than 5 within the $3 \sigma$ uncertainties is found even with the isotropic angular emissivity. The limb darkening is responsible for the further steepening. Figure 8 shows a contour plot between the radial emissivity and the spin. In contrast 


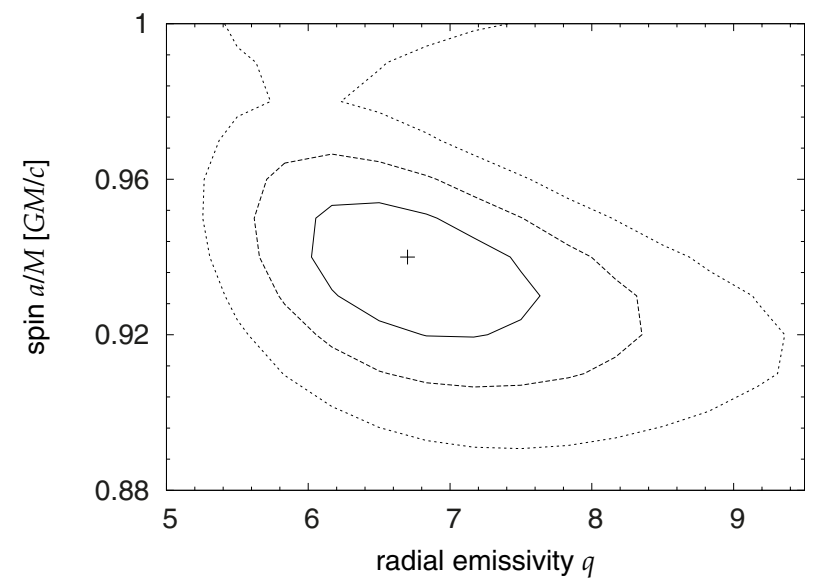

Fig. 8. Contour plot of the radial emissivity parameter $q$ and the spin $a$. The data were created by a lamp-post model with the height $h=1.5 r_{\mathrm{g}}$, radially stratified ionisation, and isotropic angular emissivity. A singleionisation model with limb darkening and power-law description for the radial emissivity was used to fit the data. The best-fit parameters are indicated by a small cross within the contours.

to the radial emissivity, the spin is found to be close to its default value.

\section{Discussion and conclusions}

We have addressed the steep radial emissivities that have been detected in the reflection components of the X-ray spectra of active galaxies and black-hole binaries. We investigated some possible explanations, and to this end performed several simulations to reveal the degeneracies of the radial emissivity with other parameters and the intrinsic assumptions of the relativistic reflection model. We realised that the steep radial emissivity may be explained by either (i) the geometrical properties of the disc-illuminating corona, (ii) the use of an improper model assumption about the angular directionality, or (iii) radially structured ionisation. The first puts rather extreme requirements on the corona. It needs to be very bright and occur at a very low height above the black hole. The second is likely due to use of an improper prescription for the emission directionality in the black hole accretion disc. The last one is related to a probable radial dependence of the disc ionisation, which plays a significant role in the total shape of the reflection spectrum.

\subsection{Lamp-post scenario}

The steep radial emissivity may be related to the properties of the disc-illuminating corona as suggested before by Wilms et al. (2001). The geometry of the emitting region certainly plays a significant role. A very centrally localised source at a low height above the black hole horizon would irradiate the disc mainly in its central region. The illumination in this area is greatly enhanced by the gravitational light-bending effect (Miniutti \& Fabian 2004; Niedźwiecki \& Życki 2008; Wilkins \& Fabian 2011; Dovčiak et al., in prep.).

To achieve steep radial emissivity, which is assumed to be proportional to the illumination, the source must be sufficiently close to the black hole. However, in this case the primary emission has to be extremely bright because only a small fraction would overcome the strong gravitational pull of the black hole and reach the observer (see Fig. 2 in Dovčiak et al. 2011). The importance of these effects declines sharply with height.
At heights $h \gtrsim 3 r_{\mathrm{g}}$, the radial emissivity profile is similar to the simple power-law with the standard value $(q=3)$. For even larger heights, the irradiation profile is more complicated (see Figs. 3 and 4 in Dovčiak et al. 2011). It decreases steeply only very close to the black-hole horizon, then becomes rather flat $(q<3)$ when still in the inner parts of the disc and finally reaches the standard value far from the centre.

\subsection{Angular directionality}

For the angular emissivity, the limb darkening law is frequently used. Several simulations, however, suggest that the directionality is the opposite of limb darkening (see e.g. Różańska et al. 2011, and references therein). The emission angle in the innermost region of the disc is always very high owing to strong aberration. The flux contribution from this region is therefore underestimated by models with limb darkening if the angular emissivity is indeed different. This effect could lead to an approximately $20 \%$ overestimation of either the spin or the inner radial emissivity parameter. Svoboda et al. (2009) reanalysed the XMM-Newton observation of MCG-6-30-15 and showed that the radial emissivity might be a more sensitive parameter to the angular directionality than the spin. This is especially true when the spin value itself is very high (close to one).

\subsection{Radially structured ionisation}

We also discussed the impact of the probable radial dependence of the disc surface ionisation. The disc illumination by a corona is commonly assumed to be stronger in the innermost regions. We therefore assumed that the ionisation is higher in the innermost region and decreases with radius. We did not consider other aspects that affect the ionisation structure of the disc, such as the density profile, vertical structure, and thermal processes (the last one being especially relevant for the stellar-mass black hole binaries). We simply assumed that the radial dependence of the irradiation is the dominating effect in determining the ionisation of the disc surface. This is especially true when the lamp-post scenario for a source of low height is considered.

We note, however, that the density may still play an important role around the marginally stable orbit. While the NovikovThorne profile of the density diverges there (Novikov \& Thorne $1973)$, i.e. the ionisation would decrease to zero, the accretion disc may extend below the marginally stable orbit owing to the presence of a magnetic field, as in the GRMHD simulations of Hawley \& Krolik (2006). The density does not increase to an infinite value, but instead decreases close to the marginally stable orbit (see also Reynolds \& Fabian 2008). This region was, for clarity, not shown in Fig. 3 but we plan to investigate it in more detail in a follow-up paper.

By fitting the simulated data, we realised that the radial decrease in the disc ionisation may account for the radialemissivity steepness equally as well as the assumption of the centrally localised corona. For the case of an isotropically illuminated disc, we obtained radial emissivities of $q \approx 4-5$, i.e. values similar to those for the lamp-post irradiation of a cold disc with the height $h=1.5 r_{\mathrm{g}}$. This is due to the different shapes of the reflection model for different ionisation parameters. The softer, more ionised reflection comes from the innermost part of the disc. When a simplified model with a single ionisation is used to fit the data, it may lead to a significant underestimation of the flux from the innermost regions, which is then artificially compensated for by a steep value of the radial emissivity profile in the model. 
The main difference between the single-ionisation model and the data created by a complex ionisation occurs at the iron-line energy band and its edge (see Figs. 5 and 7). While the shape of the "complex" reflection continuum may be mimicked by a modest value of the ionisation and steep radial emissivity, the iron line peak due to reflection from the cold distant parts of the disc is not incorporated in the simplified model. An additional emission line is then needed to model the residuals. In our test case, the equivalent width of such a line was found to be about $40 \mathrm{eV}$. Several AGN spectra, such as MCG-6-30-15 (Fabian et al. 2002), have been found to contain, together with the broad disc component, a narrow iron line associated with reflection from a distant torus. A typical equivalent width is $\sim 100 \mathrm{eV}$. The reflection from the outer parts of the accretion disc might provide a significant contribution to this line when the single-ionisation reflection model is applied to the disc-line modelling.

In our simulations, we considered the radial dependence of the ionisation. However, a similar effect on the emissivity profile may also be caused by a vertically structured accretion disc where the core is cold but the outer layers are hot, i.e. strongly ionised (Nayakshin et al. 2000). Nayakshin \& Kallman (2001) showed that in the case of AGNs, when the illuminating flux is much higher than the thermal radiation of the disc, the hot skin of the disc is completely ionised and most of the re-processing occurs in the disc core. However, Dauser et al. (2012) revealed the significant presence of highly ionised reflection together with relatively cold reflection from the disc in the X-ray spectrum of a narrow-line Seyfert 1 galaxy 1H0707-495. The doublereflection model is shown in their Fig. 6. Owing to their different shapes, the ionised reflection contributes more to the red wing of the observed iron line than the cold component. Neglecting the ionised component would therefore result in the measurement of a steeper radial emissivity index.

Finally, we note that the simulations with the radially changing ionisation for the isotropic irradiation were done with the assumption of a "standard" value for the radial emissivity, $q=3$. However, non-thermal coronal emission does not necessarily need to behave in the same way as the thermal dissipation of the disc. The interaction between the disc and the corona is more complicated, including the radiation and magnetic processes (see e.g. Haardt \& Maraschi 1991; Czerny \& Goosmann 2004; Goosmann et al. 2006; Różańska et al. 2011). In particular, when the magnetic field is considered, the intrinsic profile might already be as steep as $r^{-5}$ (Kawanaka et al. 2005). Further investigation of these poorly understood disc-corona interactions is therefore desirable.

\subsection{The combined effect}

All the effects that we have discussed are not independent of each other. Irradiation by a compact centrally localised source is very anisotropic (Miniutti \& Fabian 2004). The central regions are more illuminated and thus, more likely to be ionised than the outer parts of the accretion disc. The effect of a hypothetical radial stratification of the disc ionisation is stronger than in the case of isotropic irradiation. We therefore performed a final simulation including both effects together. We simulated the data with the lamp-post geometry and the ionisation stratification calculated from the theoretical irradiation profile (assuming constant density). We fitted the generated spectrum with a singleionisation model with a broken power-law for radial emissivity. As expected, we obtained significantly steeper radial emissivity, $q \approx 7$ (see the right panel of Table 2 and Fig. 8).
Angular directionality plays an important role in the total emissivity. We showed that the model with limb darkening overestimates the index of the radial emissivity profile by $5-10 \%$ (see the right panel of Fig. 7). The effect might be even stronger because our numerical calculations of local emissivity suggest that there is a limb-brightening effect, while we conservatively used isotropic directionality in our simulations. Moreover, in the case of anisotropic illumination by a lamp-post source, the emissivity also depends on the incident angle with a similar limbbrightening profile (Dovčiak et al., in prep.). The highest output of the reflected emission is thus obtained when both angles are grazing (perpendicular to the disc normal). This happens especially very close to the black hole owing to the large aberration that is caused by the extreme velocities of the matter in the inner disc. Hence, the flux from the innermost region might be significantly enhanced and thus the radial-emissivity index overestimated.

\section{Summary}

Very steep radial emissivity profiles with $q \sim 7$ have been previously claimed from the analysis of the disc reflection component in the X-ray spectra of some AGNs and black hole binaries. We have shown here that these profiles with $q \sim 4-5$ can be a natural consequence of either i) a compact centrally concentrated X-ray corona located 1-2 $r_{\mathrm{g}}$ away from the central black hole, or ii) the radial dependence of the disc ionisation state. By combining the two effects, emissivity indices as steep as $q \sim 7$ have been obtained, which strongly suggests that objects such as 1H 0707-495 and IRAS 13224-3809 are indeed dominated by a centrally concentrated X-ray corona whose radially-dependent disc irradiation induces a ionisation profile $\xi(r) \neq$ const. at the disc surface. We have also demonstrated that an additional steepening can be simply an artifact of improper usage of the emission directionality in the relativistic models.

Acknowledgements. The work was supported from the grant ME09036 in the Czech Republic (VK). J.S. and V.K. acknowledge support from the Faculty of the European Space Astronomy Centre (ESAC). G.M. thanks the Spanish Ministerio de Ciencia e Innovación and CSIC for support through a Ramón y Cajal contract. Financial support for this work was also provided by the Spanish Ministry of Science and Innovation (now Ministerio de Economá y Competitividad) through grant AYA2010-21490-C02-02.

\section{References}

Arnaud, K. A. 1996, in Astronomical Data Analysis Software and Systems V, eds. G. H. Jacoby, \& J. Barnes, ASP Conf. Ser., 101, 17

Ballantyne, D. R., Vaughan, S., \& Fabian, A. C. 2003, MNRAS, 342, 239

Ballantyne, D. R., Ross, R. R., \& Fabian, A. C. 2001, MNRAS, 327, 10

Ballantyne, D. R., McDuffie, J. R., \& Rusin, J. S. 2011, ApJ, 734, 112

Beckwith, K., \& Done, C. 2004, MNRAS, 352, 353

Bhayani, S., \& Nandra, K. 2011, MNRAS, 416, 629

Blandford, R. D., \& Znajek, R. L. 1977, MNRAS, 179, 433

Blustin, A. J., Page, M. J., Fuerst, S. V., Branduardi-Raymont, G., \& Ashton, C. E. 2005, A\&A, 431, 111

Brenneman, L. W., \& Reynolds, C. S. 2006, ApJ, 652, 1028

Brenneman, L. W., \& Reynolds, C. S. 2009, ApJ, 702, 1367

Brenneman, L. W., Elvis, M., Krongold, Y., Liu, Y., \& Mathur, S. 2012, ApJ, 744,13

Czerny, B., \& Goosmann, R. 2004, A\&A, 428, 353

Dauser, T., Svoboda, J., Schartel, N., et al. 2012, MNRAS, 422, 1914

de La Calle Pérez, I., Longinotti, A. L., Guainazzi, M., et al. 2010, A\&A, 524, A50

Dovčiak, M. 2004, Ph.D. Thesis (Prague: Charles University)

Dovčiak, M., Karas, V., \& Yaqoob, T. 2004, ApJS, 153, 205

Dovčiak, M., Muleri, F., Goosmann, R. W., Karas, V., \& Matt, G. 2011, ApJ, 731,75 
Dumont, A.-M., Abrassart, A., \& Collin, S. 2000, A\&A, 357, 823 Fabian, A. C., Vaughan, S., Nandra, K., et al. 2002, MNRAS, 335, L1 Fabian, A. C., Zoghbi, A., Ross, R. R., et al. 2009, Nature, 459, 540 Fabian, A. C., Wilkins, D. R., Miller, J. M., et al. 2012, MNRAS, 424, 217 George, I. M., \& Fabian, A. C. 1991, MNRAS, 249, 352

Ghisellini, G., Haardt, F., \& Matt, G. 1994, MNRAS, 267, 743

Goosmann, R. W., Czerny, B., Mouchet, M., et al. 2006, Astron. Nachr., 327, 977

Goosmann, R. W., Mouchet, M., Czerny, B., et al. 2007, A\&A, 475, 155

Haardt, F. 1993, ApJ, 413, 680

Haardt, F., \& Maraschi, L. 1991, ApJ, 380, L51

Hawley, J. F., \& Krolik, J. H. 2006, ApJ, 641, 103

Kawanaka, N., Mineshige, S., \& Iwasawa, K. 2005, ApJ, 635, 167

Krongold, Y., Nicastro, F., Elvis, M., et al. 2007, ApJ, 659, 1022

Laor, A. 1991, ApJ, 376, 90

Magdziarz, P., \& Zdziarski, A. A. 1995, MNRAS, 273, 837

Martocchia, A., Karas, V., \& Matt, G. 2000, MNRAS, 312, 817

Martocchia, A., Matt, G., \& Karas, V. 2002, A\&A, 383, L23

Matt, G., Perola, G. C., \& Piro, L. 1991, A\&A, 247, 25

Matt, G., Fabian, A. C., \& Ross, R. R. 1993, MNRAS, 262, 179

McClintock, J. E., \& Remillard, R. A. 2006, Black hole binaries, in: Compact Stellar X-ray Sources, eds. W. H. G. Lewin, \& M. van der Klis (Cambridge: Cambridge University Press), 157

Merloni, A., Heinz, S., \& di Matteo, T. 2003, MNRAS, 345, 1057

Miller, J. M. 2007, ARA\&A, 45, 441

Miller, J. M., Reynolds, C. S., Fabian, A. C., Miniutti, G., \& Gallo, L. C. 2009, ApJ, 697, 900

Miniutti, G., \& Fabian, A. C. 2004, MNRAS, 349, 1435

Miniutti, G., Fabian, A. C., Goyder, R., \& Lasenby, A. N. 2003, MNRAS, 344, L22

Miniutti, G., Fabian, A. C., \& Miller, J. M. 2004, MNRAS, 351, 466

Miniutti, G., Fabian, A. C., Anabuki, N., et al. 2007, PASJ, 59, 315

Nandra, K. 2011, in The X-ray Universe 2011, eds. J.-U. Ness, \& M. Ehle, 22
Nandra, K., O’Neill, P. M., George, I. M., \& Reeves, J. N. 2007, MNRAS, 382, 194

Nayakshin, S., \& Kallman, T. R. 2001, ApJ, 546, 406

Nayakshin, S., Kazanas, D., \& Kallman, T. R. 2000, ApJ, 537, 833

Niedźwiecki, A., \& Życki, P. T. 2008, MNRAS, 386, 759

Novikov, I. D., \& Thorne, K. S. 1973, in Black Holes (Les Astres Occlus), 343

Penna, R. F., McKinney, J. C., Narayan, R., et al. 2010, MNRAS, 408, 752

Ponti, G., Gallo, L. C., Fabian, A. C., et al. 2010, MNRAS, 406, 2591

Reynolds, C. S., \& Begelman, M. C. 1997, ApJ, 488, 109

Reynolds, C. S., \& Fabian, A. C. 2008, ApJ, 675, 1048

Reynolds, C. S., \& Nowak, M. A. 2003, Phys. Rep., 377, 389

Reynolds, C. S., Brenneman, L. W., Wilms, J., \& Kaiser, M. E. 2004, MNRAS, 352, 205

Ross, R. R., \& Fabian, A. C. 1993, MNRAS, 261, 74

Ross, R. R., \& Fabian, A. C. 2005, MNRAS, 358, 211

Różańska, A., Dumont, A.-M., Czerny, B., \& Collin, S. 2002, MNRAS, 332, 799

Różańska, A., Madej, J., Konorski, P., \& Sadowski, A. 2011, A\&A, 527, A47

Shakura, N. I., \& Sunyaev, R. A. 1973, A\&A, 24, 337

Shapiro, S. L., Lightman, A. P., \& Eardley, D. M. 1976, ApJ, 204, 187

Svoboda, J., Dovčiak, M., Goosmann, R., \& Karas, V. 2009, A\&A, 507, 1

Svoboda, J., Guainazzi, M., \& Karas, V. 2010, A\&A, 512, A62

Tombesi, F., Cappi, M., Reeves, J. N., et al. 2010, A\&A, 521, A57

Vaughan, S., \& Fabian, A. C. 2004, MNRAS, 348, 1415

Wilkins, D. R., \& Fabian, A. C. 2011, MNRAS, 414, 1269

Wilkins, D. R., \& Fabian, A. C. 2012, MNRAS, 424, 1284

Wilms, J., Reynolds, C. S., Begelman, M. C., et al. 2001, MNRAS, 328, L27

Zhang, S. N., Cui, W., \& Chen, W. 1997, ApJ, 482, L155

Zhou, X. L., Zhao, Y. H., \& Soria, R. 2011, MNRAS, 413, L61

Zoghbi, A., Fabian, A. C., Uttley, P., et al. 2010, MNRAS, 401, 2419

Życki, P. T., \& Czerny, B. 1994, MNRAS, 266, 653

Zycki, P. T., Krolik, J. H., Zdziarski, A. A., \& Kallman, T. R. 1994, ApJ, 437, 597 